\title{
5G Communication Based Distributed Fault Recovery Scheme of Active Distribution Network
}

\author{
Xin Li, Houlei Gao, Tong Yuan, Bin Xu \\ Key Laboratory of Power System Intelligent Dispatch and Control of Ministry of Education, Shandong University, Jinan, Shandong, \\ 250061, China
}

\begin{abstract}
As more and more distributed power sources are connected to low and medium voltage distribution networks, the traditional single-ended passive distribution networks have evolved into multiterminal, multi-source active distribution networks. When distributed generations with high permeability are connected to a distribution network, the structure and power flow of this network will change significantly, thus the original fault detection method and reclosing scheme may be challenged, which may cause incorrect action of protection or failure of reclosing. On basis of that, this paper proposes an active distribution network fault recovery scheme based on $5 \mathrm{G}$ wireless communication, in which the topology recognition technology and smart terminal units with peer-to-peer communication capability are applied. To prove the method's feasibility, delay of $5 \mathrm{G}$ communication is analysed and tested online. In addition, a model of $10 \mathrm{kV}$ active distribution network is built on Real Time Digital Simulation system. Principle investigation and simulation indicate that the proposed scheme can adapt to the change of network structure and implement the fault self-healing quickly.
\end{abstract}

\section{Introduction}

In early days, to clear the fault quickly and reliably in active distribution network, it is required that the distributed generator (DG) should be out of operation after fault. With the deepening of research, some scholars have put forward measures, such as limiting DG access capacity and the fault current at DG access point [1-2]. However, these methods cannot adapt to the access of DG with high permeability. Therefore, many new protection and control schemes of active distribution network have emerged. [3] took the low voltage ridethrough ability of the inverter DG into account and made the inverter DG equivalent to a voltage-controlled current source, then gave an accurate analysis and calculation method of fault current. [4] analysed the current response of inverter DG under different control modes (voltage type and current type). Based on the output current characteristics of DG, [5] proposed a distributed regional longitudinal protection scheme for distribution networks, focusing on an online associated area determining method for longitudinal protection, which can be used when the operating mode or network topology changes. This method can adaptively identify the topology and reconfigure the associated area.

With the increasing proportion of $D G$ in the distribution network, the topology and operation mode of distribution network have undergone great changes. The previous methods used for fault recovery cannot be directly applied to the distribution network with high permeability [6]. During the active distribution network supply restoration, the influence of DG must be taken into consideration [7].

To achieve fault recovery, the centralized control mode or local control mode are generally adopted by current distribution network. Both the "centralized" and "local" control modes still have deficiencies, such as long outage time, imperfect protection scheme and inflexible control method. With the development of communication and smart terminal technology, distributed control method, which is not dependent on master station, gradually becomes a trend of the development of distribution network [8].

At present, distributed topology identification and fault recovery scheme based on fibre-optical communication are used in distribution networks widely. But in many areas, it is difficult or even impossible to lay optical cables. To a certain extent, this problem seriously limits the application of distributed control scheme in distribution network. Compared to fibre-optical communication system, 5G wireless communication network provides a much more flexible and economical alternative [9]. On one hand, the 5G communication technology has great flexibility and adaptability, hence DG can conveniently access to the protection and control system of existing distribution network. On the other hand, the wireless commutation network is with the advantages of flexible networking, low infrastructure cost and short construction period, which are not found

*Corresponding author's e-mail: houleig@sdu.edu.cn 
in fibre-optical communication mode. Based on the Ultra-reliable and Low Latency Communications (URLLC) scenario, the $5 \mathrm{G}$ wireless communication technology can establish a peer-to-peer communication network conveniently, which can meet the requirement for low-latency and high-reliability data exchange between two or more STUs in distribution system. 5G communication technology is more than a simple upgrade of $4 \mathrm{G}$ communication. Unlike the one size fits all $4 \mathrm{G}$ core networks, the $5 \mathrm{G}$ core network must be flexible and adaptable and is expected to simultaneously provide optimized support for the diverse $5 \mathrm{G}$ use case categories [10].

\section{Recognition of distribution network topology information}

The STU nearby power source is a protection unit with the ability of processing data and controlling switches autonomously. These STUs are able to achieve peer-topeer communication via fibre-optical or $5 \mathrm{G}$ networks. Distribution network is with various network topologies for different operating modes. Therefore, STU is required for the ability to obtain topology information, which can be divided into static and dynamic types.

For static network topology information, it includes the information about the local STU and adjacent STUs. Such information needs to be manually configured and does not need to be changed frequently. After changing the structure of network, the corresponding information needs to be modified manually by staffs. The maintenance is less and simple, because the topology information configured in a STU is partial information only. For dynamic topology information, the corresponding information should be automatically updated with the change of topology structure or operating mode.

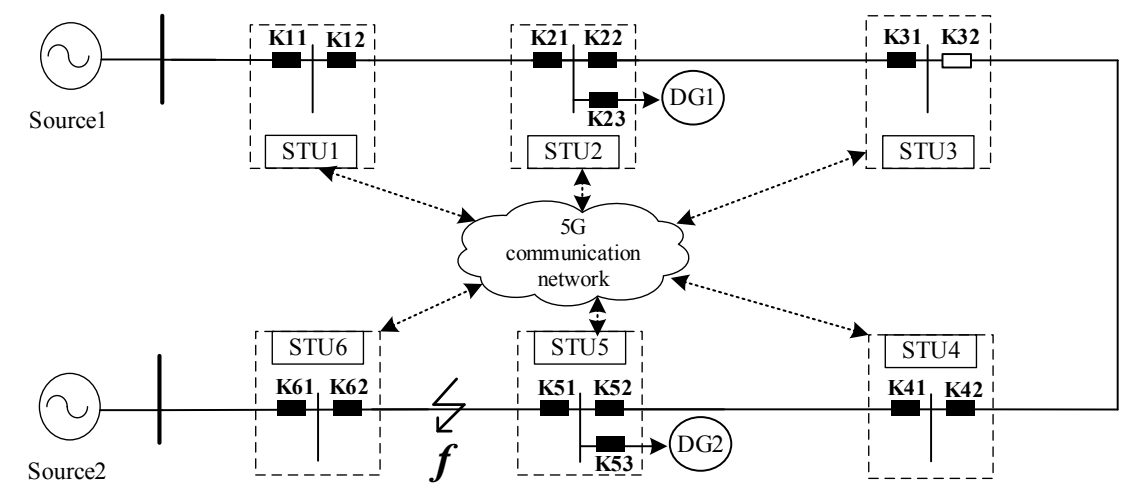

Figure 1. Active distribution network equipped with STUs.

In an active distribution network equipped with STUs, as shown in Figure 1, the process of acquiring dynamic network topology information via $5 \mathrm{G}$ communication network is given as follows:

a) The power-side STU1 sends a switch status query instruction to its neighbouring STU2.

b) STU2 marks the upstream STU for the STU1 that sends instructions to it and the downstream STU for another adjacent unit.

c) Since both K21 and K22 are closed states, STU2 forwards the switch status query instruction to downstream STU3.

d) Similarly, STU6 forwards the switch status query instruction downstream step by step.

e) When STU3 receives the query directives forwarded by STU2 and STU4, it can confirm that it is STU at the contact switch according to the switching state of K32.

\section{Distributed fault recovery scheme of active distribution network}

Most high-level functions of $5 \mathrm{G}$ base station are concentrated in the Central Office (CO) of data centre, some wireless-related functions are managed by the Central Unit (CU), and the underlying functions of base station are implemented by the Distributed Unit (DU). To meet the delay requirement of uRLLC service, the corresponding CU should be deployed with DU in the same place. Massive MIMO (mass multiple input and output) technology is introduced into $5 \mathrm{G}$ communication technology, hence the traditional Remote Radio Unit (RRU) and antenna are combined into the Active Antenna Unit (AAU). 


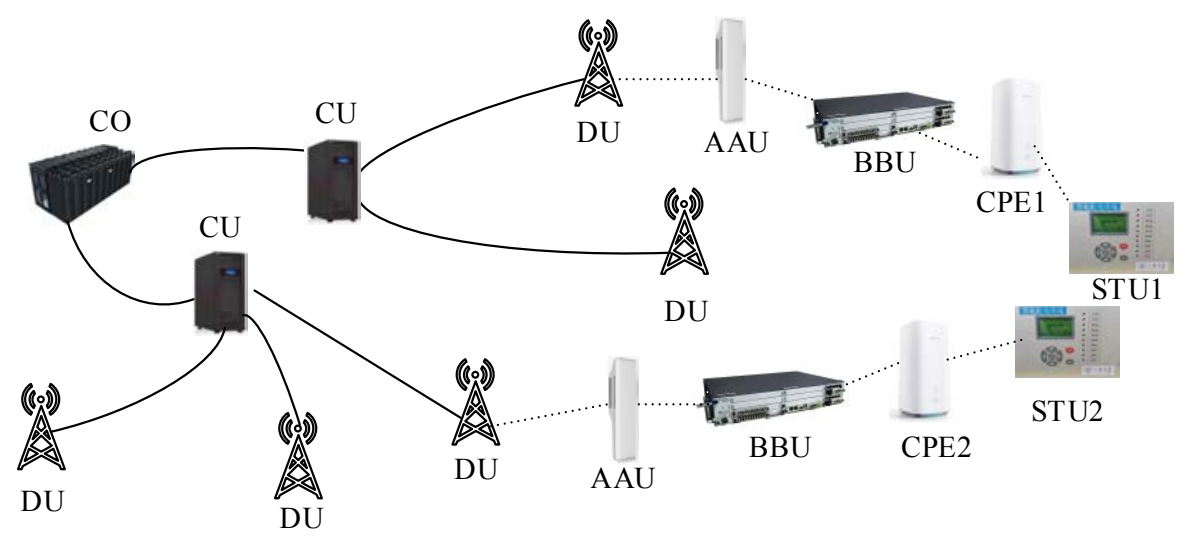

Figure 2. Communication link diagram of $5 \mathrm{G}$ network.

It can be seen from Figure 2 that STUs access 5G core network via the Customer Premise Equipment (CPE), Building Base Band Unite (BBU) and AAU to receive and send data. In power system, after the communication infrastructure is completed, STU can access the communication network with the help of $5 \mathrm{G}$ CPE only. When there are many STUs in a distribution system, each STU should be equipped with a CPE, to access the $5 \mathrm{G}$ communication network and construct a peer-to-peer communication network. With the advancement of technology, the price of CPE will be lower, and the degree of integration will be higher. In the future, CPE is expected to be integrated in STU, thus the installation and network access of STU will be more economical and easy to implement.

Based on the topology identification, this paper proposes a distributed fault recovery scheme for active distribution network. For the fault at $f$ (as shown in Figure 2), the fault isolation process is as follows:

a) STU5 and STU6 send an instruction to request the current to each other;

b) STU5 sends the current data flowing through switch K51 to STU6, and STU6 sends the current data flowing through switch K62 to STU5;

c) STU5 and STU6 detect the fault section respectively, using the differential and amplitude comparison criteria;

d) Trip signals are delivered to breakers K51 and K62 from STU5 and STU6, respectively;

e) The breakers operate and the fault can be isolated.

After the fault section is removed, STU6, the upstream STU of the section, begins recovery from malfunction. The specific recovery process is as follows:

First, STU6 checks for no voltage to ensure that the downstream circuit breaker is opened. If there is no voltage on the line, STU6 will issue a reclosing command to $\mathrm{K} 62$.
Next, there are two situations. If the fault is permanent, STU6 detects an overcurrent signal and the breaker K62 trips again. Meanwhile, STU6 will inform STU3 at the contact switch that the reclosing has failed. STU3 will control the closing of $\mathrm{K} 32$ on the premise that the closing conditions are met and realize the restoration of power supply in non-fault section. If the fault is transient, STU6 informs STU5 of successful reclosing. Supposed downstream DG2 has quit operation, STU5 can directly issue closing instructions to K51 to complete the entire reclosing process. Or supposed DG2 is still in operation, there is voltage at the downstream feeder. To prevent the large impulse current generated during the grid-connection, STU5 needs to check synchronous closing.

Finally, the power loss area is restored, and the fault recovery is completed.

\section{Case study}

In $5 \mathrm{G}$ communication environment, the delay is tested by the Packet Internet Groper (PING) command based on TCP/IP protocol. Ping is a service command that works in the application layer of TCP/IP network architecture. It is mainly used to send Internet Control Message Protocol (ICMP) Echo request Message to a specific destination host. The round-trip time of the data packet can be approximately considered as the time delay on the communication link.

The communication link between two STUs is shown in Figure 2. The test result on the site is shown in Figure 3. The maximum of communication delay for round-trip communication between STU1 and STU2 is $26 \mathrm{~ms}$, the minimum is $18 \mathrm{~ms}$, and the average is about $20.62 \mathrm{~ms}$. Since the result of the test is round-trip delay, it can be roughly considered that the one-way delay is half of the round-trip delay. 


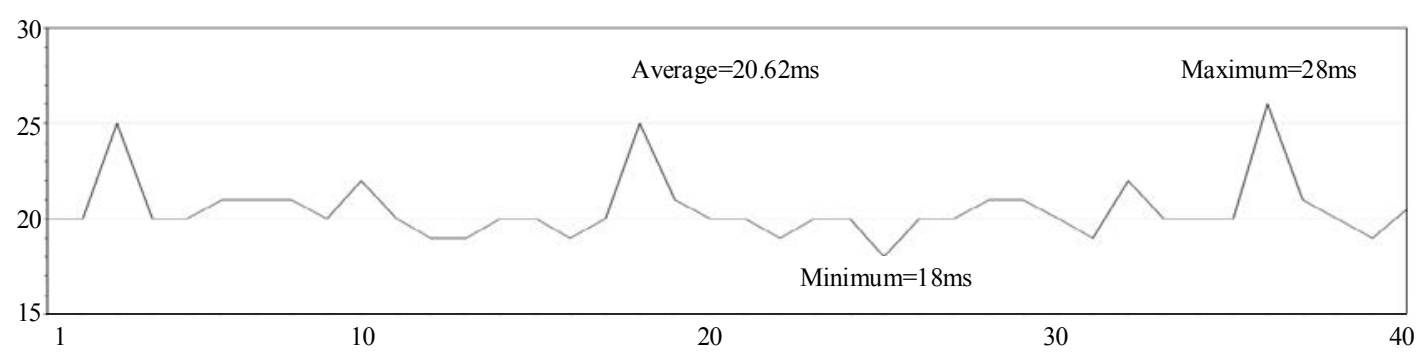

Figure 3. 5G communication delay test based on PING instruction.

To verify the accuracy and reliability of the proposed fault recovery scheme, the simulation model in Figure 4 was built in Real-time Digital Simulation (RTDS) system. The analog signal generated in RTDS is sent into the corresponding STU after passing through the amplifier. After the switch signal is output from RTDS, it is connected to the telecommunicating interface of corresponding STU through the relay. The foregoing is to simulate the operation of a real power distribution system. To make the proposed scheme have better portability in $5 \mathrm{G}$ wireless communication network, the information exchange among STUs adopts peer-to-peer communication. In the model shown in Figure 4, the power capacity is set at $10 \mathrm{MW}$, the load connected by each bus is $2 \mathrm{MW}$. Red means the breaker is in position, green means it is off. The specific system model parameters are shown in Table 1 and Table 2.

Table 1. System model parameters table.

\begin{tabular}{ccc}
\hline Element & \multicolumn{1}{c}{ Project } & Parameter \\
\hline \multirow{2}{*}{ Source1,2 } & Voltage & $10 \mathrm{kV}$ \\
\cline { 2 - 3 } & Impedance & $1 \angle 80^{\circ}$ \\
\cline { 2 - 3 } DG1,2 & Type & Inverter \\
\cline { 2 - 3 } & Control strategy & PQ control \\
\cline { 2 - 3 } & Power & 2MW \\
\hline \multirow{2}{*}{ The load on the bus } & Capacity & $2 \mathrm{MW}$ \\
\cline { 2 - 3 } & Power factor & 0.9 \\
\hline
\end{tabular}

Table 2. Line parameters table.

\begin{tabular}{|c|c|c|}
\hline \multirow{3}{*}{$\begin{array}{l}\text { Impedance } \\
\text { parameter }\end{array}$} & $\begin{array}{c}\text { Positive sequence } \\
\text { impedance }\end{array}$ & $(0.17+0.34 \mathrm{j}) \Omega$ \\
\hline & $\begin{array}{c}\text { negative sequence } \\
\text { impedance }\end{array}$ & $(0.17+0.34 \mathrm{j}) \Omega$ \\
\hline & $\begin{array}{c}\text { zero sequence } \\
\text { impedance }\end{array}$ & $(0.42+1.51 \mathrm{j}) \Omega$ \\
\hline length of line & \multicolumn{2}{|c|}{$2 \mathrm{~km}$} \\
\hline
\end{tabular}
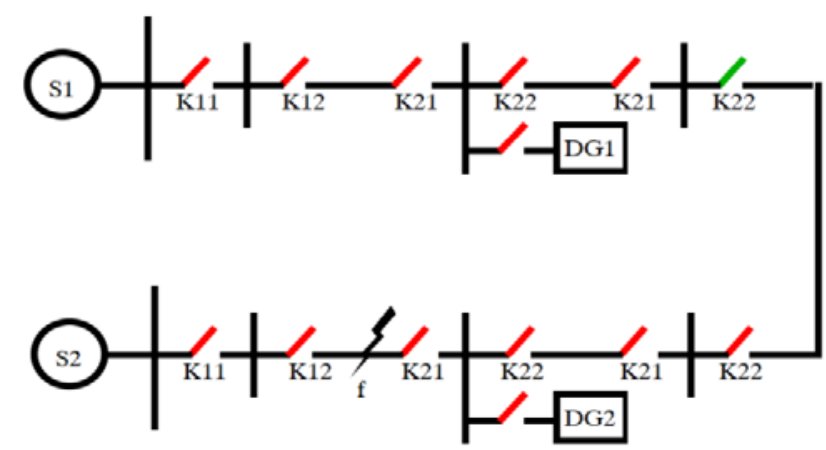

Figure 4. Simulation model.

When a permanent three-phase short-circuit fault occurs at $f$, and the fault recording diagram is shown in Figure 5. 


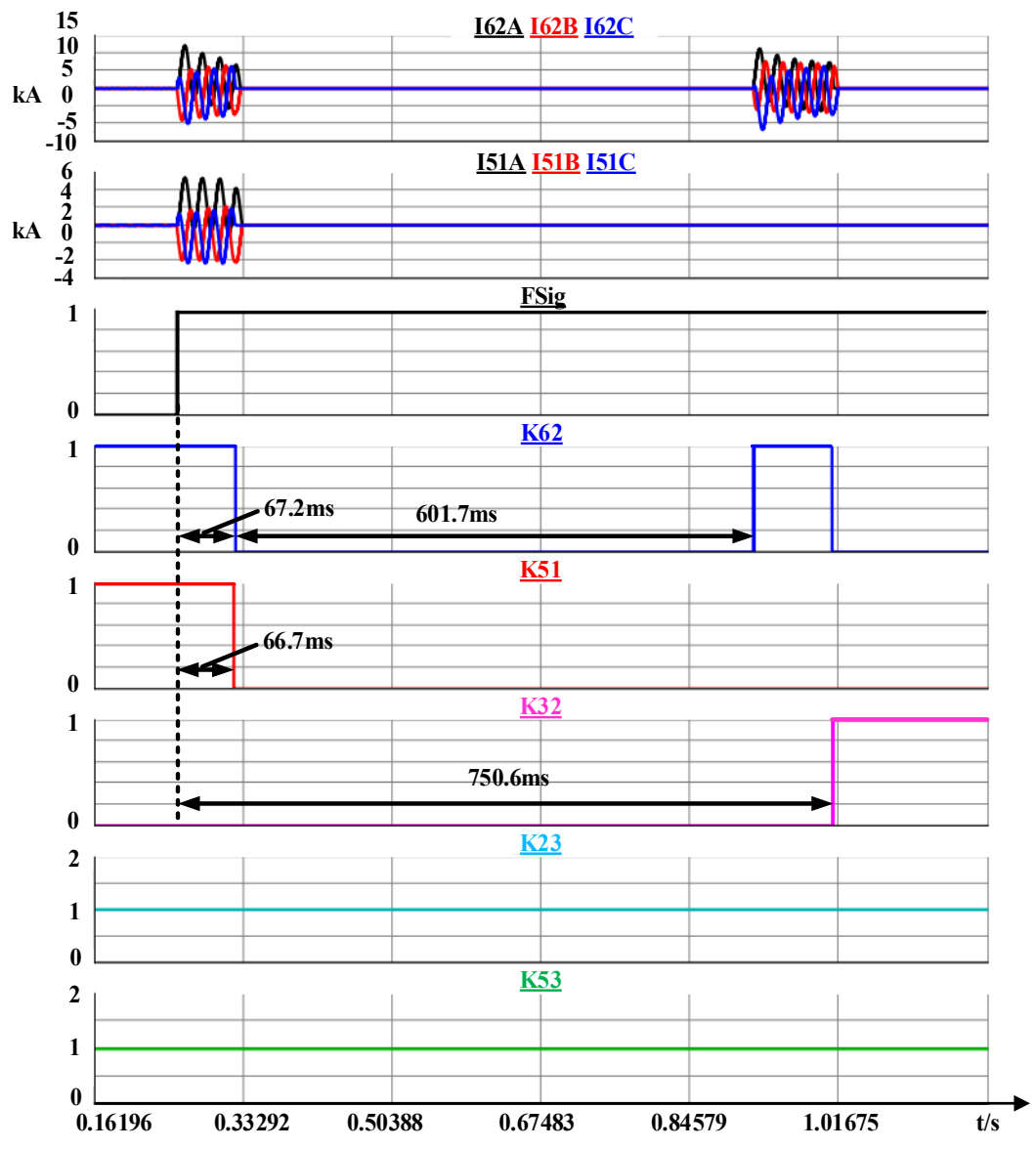

Figure 5. Recording in case of permanent fault at $f$

It can be seen from Figure 5 that about $67 \mathrm{~ms}$ after the fault, STU5 and STU6 send out the signals to trip K51 and K62 almost simultaneously. After a period of time delay (set to 600ms), STU6 first controls the circuit breaker K62 to reclose. Since the fault did not disappear, K62 failed to reclose, and STU6 immediately notified STU3 to close the switch K32 to restore power to the non-faulty section.

\section{Conclusion}

For active distribution network, this paper briefly introduces the process of identifying the topology information of the distribution network. Using the smart terminal unit, a fault recovery scheme for the active distribution network based on $5 \mathrm{G}$ communication is designed. After verification on the RTDS simulation platform, this scheme can isolate the fault fast, and restore power to the non-fault section within $1 \mathrm{~s}$, which is helpful to improve the reliability of power supply.

\section{Acknowledgments}

The work was supported by the project named "Research on key technology of multi parameter relay protection based on $5 \mathrm{G}$ communication".

\section{References}

1. Tao shun, Guo Jing, Xiao Xiangning. Analysis on Allowed Penetration Level of Distributed Generation and Its Grid-Connected Position Based on Principles of Current Protection[J]. Power System Technology. 2012, 36(01):265-270.

2. Fu Wenxiu, Fan Chunju, Yang Lian, et al. Locating and sizing of distributed generators and current protection strategy in distribution networks[J]. Automation of Electric Power Systems. 2014,38(10):78-84.

3. Wang Gang, Li Haifeng, Pan Guotao, Gao Xiang. Fault Characteristics Analysis of Distribution Networks Considering Control Scheme of Inverter Interfaced Distributed Generation[J]. Automation of Electric Power Systems, 2012,36(18):92$96+108$.

4. Baran M E, El-Markaby I. Fault analysis on distribution feeders with distributed generators[J]. IEEE Transactions on Power Systems, 2005, 20(4):1757-1764.

5. Cong Wei, Fang Fanxiu, Shi Fangfang, Lu Qingdong. An Online Associated Area Determining Method for Distributed Regional Longitudinal Protection in Smart Distribution 
Grid[J]. Automation of Electric Power Systems, 2014,38(12):67-73+111.

6. Zhang Qingjie, Lu Yuping. A New Distributed Protection Based on Adaptive Division for Relevant Fault Area[J]. Automation of Electric Power Systems, 2008(07):39-43+52.

7. Dong Xuzhu, Huang Shaoyuan, Chen Rouyi, Li Peng, Zhang Wenfeng. Self healing control technology for smart distribution system.

8. Wu Yuehua, Gao Houlei, $\mathrm{Xu}$ Bin, Kang Gengqiang, Wu Zhigang, Wang Ning. Distributed Fault Self_healing Scheme and Its
Implementation for Active Distribution Network[J]. Automation of Electric Power Systems, 2019, 43(09):181-196.

9. Hassebo A, Mohamed A A, Dorsinville R, et al. 5G-based Converged Electric Power Grid and ICT Infrastructure[C]// 2018 IEEE 5G World Forum (5GWF). IEEE, 2018.

10. Shafi M, Molisch A F, Smith P J, et al. 5G: A Tutorial Overview of Standards, Trials, Challenges, Deployment, and Practice[J]. IEEE Journal on Selected Areas in Communications, 2017, PP (99):1-1. 